\title{
Rational permutation modules for finite groups
}

\author{
Ian Hambleton ${ }^{1}$, Laurence R. Taylor ${ }^{2}$ \\ 1 Department of Mathematics \& Statistics, McMaster University, Hamilton, Ontario L8S \\ 4K1, Canada (e-mail: ian@icarus.Math.McMaster.ca) \\ 2 Department of Mathematics, University of Notre Dame, Notre Dame, IN 46556, USA \\ (e-mail: taylor.2@nd.edu)
}

Received March 17, 1997; in final form May 6, 1998

A rational permutation module for a finite group $G$ is a rational representation of the form $V \cong \mathbb{Q} X$ for some finite $G$ set $X$. Let $P(G)$ denote the subring of the rational representation ring $R(G)$ spanned by the permutation modules. Alternatively, $P(G)$ is the image of the Burnside ring of $G$ in $R(G)$. Define the functor $C(G)$ as the cokernel

$$
0 \longrightarrow P(G) \longrightarrow R(G) \longrightarrow C(G) \longrightarrow 0 \text {. }
$$

By the Artin Induction theorem, $C(G)$ is a finite abelian group with exponent dividing the order of $G$.

Some work on this sequence has already been done. In [14] and [16], Ritter and Segal proved that $C(G)=0$ for $G$ a finite $p$-group. Serre [17, p. 104] remarked that $C(G) \neq 0$ for $G=\mathbb{Z} / 3 \times Q_{8}$ (the direct product of a cyclic group of order 3 and a quaternion group of order 8 ).

Berz [2] gave a nice description of $P(G)$ for $G$ metabelian or supersolvable. To describe the result, recall that $R(G)$ additively is a free abelian group with basis given by the irreducible rational representations of $G$. The subgroup $P(G)$ is generated by the induced representations $\operatorname{Ind}^{G}\left(1_{H}\right)=$ $\mathbb{Q}[G / H]$, where $H$ runs over the subgroups of $G$. If $a_{\phi}$ denotes the gcd over all $H$ of the numbers $\left\langle\phi, \operatorname{Ind}^{G}\left(1_{H}\right)\right\rangle$, then $a_{\phi}$ divides $\langle\phi, \chi\rangle$ whenever $\chi$ is a virtual permutation representation. Let $\alpha_{\phi}=\frac{a_{\phi}}{\langle\phi, \phi\rangle}$.

Theorem: (Berz [2]) For $G$ metabelian or supersolvable the lattice $P(G) \subseteq$ $R(G)$ has a basis $\alpha_{\phi} \cdot \phi$ where $\phi$ runs over the irreducible rational representations of $G$.

The authors wish to thank the Max Planck Institut für Mathematik in Bonn for its hospitality and support. This research was also partially supported by NSERC and the NSF. 
It follows immediately from the definition that $P(G) \subseteq \bigoplus \alpha_{\phi} \cdot \phi$ for any finite group $G$. In an earlier version of this paper we claimed that equality held for all finite groups, but Berz [2] gives a counterexample. The error lay in the assertion that the lattice defined as $\bigoplus \alpha_{\phi} \cdot \phi$ has good induction and restriction properties.

In $\S 1$ we review some of the foundational work of A. Dress on induction theory and observe that hyperelementary computation follows for the Mackey functors $P(G), R(G)$ and $C(G)$. Since hyperelementary groups are supersolvable, Berz's result applies and this information leads in principle to further information about $C(G)$ for general groups $G$.

In $\S 2$, we prove that the functors, $P, R$ and $C$ are "detected" by the basic subquotients of $G$ [9]. This leads to a different proof of the Berz equality $P(G)=\bigoplus \alpha_{\phi} \phi$, for $G$ hyperelementary, and to more efficient methods for computing $C(G)$.

Each basic group $B$ is $p$-hyperelementary for some prime $p$ and each basic group has a unique irreducible faithful rational representation $\rho_{B}$. Representations have induction and restriction for quotient maps as well as subgroups. Hence they also have "push forward" and "pull back" maps for subquotients. If $H$ is a subquotient of $G$, we call the map from $R(G)$ to $R(H)$ the restriction and we call the map from $R(H)$ to $R(G)$ the induction map.

Hyperelementary computation and basic detection can be combined (see $\S 3)$ to give an explicit numerical criterion for an arbitrary rational representation to be a virtual permutation representation.

Theorem A: Given a rational representation $\chi$ on $G, \chi$ is a virtual permutation representation if and only if $a_{\rho_{B}}$ divides $\left\langle\chi\right.$, $\left.\operatorname{Ind}^{G}\left(\rho_{B}\right)\right\rangle$ for all basic subquotients $B$ of $G$.

In $\S 4$ we describe the basic groups and give a partial calculation of the $a_{\rho_{B}}$. In conjunction with the general theory, this leads to a short proof of the Ritter-Segal theorem in $\S 6$.

In $\S 7$ we construct examples of groups $G$ for which $C(G)$ is arbitrarily complicated. In $\S 8$ we give some calculations of $C(G)$ and prove some vanishing results. One consequence of Corollary 8.3 is:

Theorem B: If $p$ is the largest prime dividing the order of $G$, then $C(G)$ is $p$-torsion free.

To state the calculation for $G$ nilpotent (see $\$ \S 9-10$ ) we need some notation. Let $C h_{\mathbb{Q}}(G)$ denote the ring of rational valued characters of $G$, and recall that

$$
0 \rightarrow R(G) \rightarrow C h_{\mathbb{Q}}(G) \rightarrow \bigoplus \mathbb{Z} / m_{\phi} \rightarrow 0
$$

is a short exact sequence where the sum runs over the irreducible rational representations $\phi$ of $G$ and $m_{\phi}$ is the Schur index of an irreducible complex 
constituent of $\phi$. If we let $\bar{C}(G)$ denote the cokernel of the inclusion $P(G) \subseteq$ $C h_{\mathbb{Q}}(G)$, then we also have the following isomorphisms

$$
\bar{C}(G) / C(G) \cong C h_{\mathbb{Q}}(G) / R(G) \cong \bigoplus \mathbb{Z} / m_{\phi} .
$$

Let $\widetilde{R}(G)$ denote the kernel of the restriction map $R(G) \rightarrow R(\{e\})$. For nilpotent groups, $G$ is the direct sum of its $p$-Sylow subgroups $G_{p}$, and we may write $G=G_{2} \times G_{\text {odd }}$. Note that $R\left(G_{\text {odd }}\right)=\bigotimes_{p \text { odd }} R\left(G_{p}\right)$. In [14, Satz 3] it was asserted that $C(G)=0$ for $G$ nilpotent, but $C(G)=\mathbb{Z} / 2$ for $G=\mathbb{Z} / 3 \times Q_{8}$ ([14, Hilfsatz 6.1(1)] is incorrect). More generally, $C(G)=\mathbb{Z} / 2$ for $G=\mathbb{Z} / p \times Q_{2^{r}}$ for any odd prime $p$ and $r \geq 4$. On the other hand, $C(G)=0$ for $G=\mathbb{Z} / 7 \times Q_{8}$. This dependence, both on the prime factors of $G_{o d d}$ and on the quaternion algebras, complicates the calculation for nilpotent groups.

Call an odd prime $p$ non-split if $2^{w} \equiv 1(\bmod p)$ when $p-1=2^{s} w$ with $w$ odd: otherwise call it split. Let $G_{s}$ denote the product of the $p$ Sylow subgroups for split primes and let $G_{n s}$ denote the product of the $p$-Sylow subgroups for non-split primes. We will show in Proposition 6.2 that $\bar{C}\left(G_{2}\right)$ is a $\mathbb{Z} / 2$ vector space with one $\mathbb{Z} / 2$ for each irreducible rational representation whose division algebra is quaternionic. There is a direct sum decomposition $\bar{C}\left(G_{2}\right)=\bar{C}\left(G_{2}\right)_{8} \oplus \bar{C}\left(G_{2}\right)_{\geq 16}$ depending on whether the center field is $\mathbb{Q}$ or not. Then

Theorem C: For $G$ nilpotent, $\bar{C}(G)=R\left(G_{\text {odd }}\right) \otimes \bar{C}\left(G_{2}\right)$ and

$$
C(G)=\widetilde{R}\left(G_{\text {odd }}\right) \otimes \bar{C}\left(G_{2}\right)_{\geq 16} \oplus \widetilde{R}\left(G_{s}\right) \otimes R\left(G_{n s}\right) \otimes \bar{C}\left(G_{2}\right)_{8} .
$$

In this formula, the term $\widetilde{R}\left(G_{s}\right) \otimes R\left(G_{n s}\right)$ is just the kernel of the restriction map $R\left(G_{\text {odd }}\right) \rightarrow R\left(G_{n s}\right)$.

There is another description of the answer for $G$ nilpotent. Let $\mathcal{E}_{G}$ denote the set of conjugacy classes of odd order cyclic subgroups of $G$. Each $E \in \mathcal{E}_{G}$ has a unique faithful, irreducible, rational representation $\rho_{E}$. Let $m_{\mathbb{R}}(\xi)$ denote the real Schur index of an irreducible complex constituent of $\xi$, and let $f_{E}=\left[\widehat{\mathbb{Q}}_{2}\left(\rho_{E}\right): \widehat{\mathbb{Q}}_{2}\right]$.

Theorem $\mathbf{C}^{\prime}$ : For $G$ nilpotent,

$$
C(G) \cong \underset{(\xi, E)}{\bigoplus}\left\{\mathbb{Z} / 2 \mid m_{\mathbb{R}}(\xi)=2 \text { and } f_{E} \cdot[\mathbb{Q}(\xi): Q] \equiv 0(\bmod 2)\right\} .
$$

where $\xi$ runs over the irreducible rational representations of $G_{2}$ and $\{e\} \neq$ $E \in \mathcal{E}_{G}$. 
To compare the two versions, note that the rank of $R\left(G_{\text {odd }}\right)$ is just the cardinality of the set $\mathcal{E}_{G}$ (see $\S 9$ for an idempotent description of this correspondence). The condition $m_{\mathbb{R}}(\xi)=2$ picks out the quaternionic representations of $G_{2}$, the degree $[\mathbb{Q}(\xi): Q]$ is the degree of the center field extension, and $f_{E}$ determines whether $E$ is split or non-split.

Finally we remark that hyperelementary calculation has some limitations. For example, all the irreducible complex representations of the symmetric groups $\Sigma_{n}$ come from permutation modules [11, Thm.2.2.10,p.39], so $C\left(\Sigma_{n}\right)=0$. However, any finite collection of hyperelementary subgroups occurs in a fixed $\Sigma_{n}$ once $n$ is sufficiently large.

\section{A review of Dress's work on induction}

The work to which we are referring (see [4] and [5]) assumes that we are given a Mackey functor $\mathcal{M}$ and a family of subgroups of $G$, denoted $\mathcal{H}$. In general it is only important that $\mathcal{H}$ be closed under conjugation and subgroups, but in this paper it is the family of hyperelementary subgroups.

One can then form what Dress calls an Amitsur complex: this is a chain complex

$$
\mathcal{M}(G) \stackrel{\partial_{0}}{\longrightarrow} \bigoplus_{H \in \mathcal{H}} \mathcal{M}(H) \stackrel{\partial_{1}}{\longrightarrow} \ldots
$$

where the higher terms are explicitly described sums of $\mathcal{M}$ applied to elements of $\mathcal{H}$. The boundary map $\partial_{0}$ is the sum of restriction maps and the higher $\partial_{i}$ are just sums and differences of restriction maps. There is a second Amitsur complex defined using induction maps for which the boundary maps go the other direction.

Dress further assumes that some Green ring, say $\mathcal{G}$, acts on $\mathcal{M}$. Write

$$
\delta_{\mathcal{G}}^{\mathcal{H}}: \bigoplus_{H \in \mathcal{H}} \mathcal{G}(H) \rightarrow \mathcal{G}(G)
$$

for the sum of the induction maps.

Theorem 1.1: If there exists $y \in \bigoplus_{H \in \mathcal{H}} \mathcal{G}(H)$ such that $\delta_{\mathcal{G}}^{\mathcal{H}}(y)=1 \in$ $\mathcal{G}(G)$, then both Amitsur complexes for $\mathcal{M}$ are contractable.

Remark: One writes the conclusion as $\mathcal{M}(G)=\lim _{\mathscr{H}} \mathcal{M}(H)$ or $\mathcal{M}(G)=$ $\lim _{\overrightarrow{\mathcal{H}}} \mathcal{M}(H)$ where the first limit made up of restrictions and the second of inductions. The result above follows from [5, Prop.1.2,p.305] and the remark just above [5, Prop.1.3,p.190].

This is a very powerful theorem whose main difficulty in use comes in finding a Green ring which acts. The Burnside ring is a Green ring which 
always acts on any Mackey functor, but it satisfies Dress's condition on $\delta_{\mathcal{G}}^{\mathcal{H}}$ if and only if $G \in \mathcal{H}$.

Observation: The image of the Burnside ring in $\mathcal{G}$, denoted $\mathcal{A}_{\mathcal{G}}$, is a Green ring which acts on $\mathcal{M}$. The method that proved $\delta_{G}^{\mathcal{H}}$ hits 1 will probably prove that $\delta_{\mathcal{A}_{\mathcal{G}}}^{\mathcal{H}}$ also hits 1. The advantage of $\mathcal{A}_{\mathcal{G}}$ over $\mathcal{G}$ is that $\mathcal{A}_{\mathcal{G}}$ acts on Mackey functors which are subfunctors or quotient functors of $\mathcal{M}$ whereas $\mathcal{G}$ may not act on all of them. In particular, $\mathcal{G}$ never acts on $\mathcal{A}_{\mathcal{G}}$ unless they are equal.

This observation has been made before, e.g. [12, p.253], [10, Sect. 3], and [1]. For $\mathcal{G}$ the complex representation ring, Dress [4, Prop. 5.2,p. 210] proved that $\delta_{\mathcal{G}}^{\mathcal{H}}$ hits 1 . The same proof applies verbatim to $P(G)$, the image of the Burnside ring in $R(G)$. It follows that

Proposition 1.2: Any subquotient-Mackey functor of the complex representation ring has hyperelementary calculation.

Remark: A subquotient-Mackey functor is a sub-Mackey functor followed by a quotient Mackey functor. Examples include $P, C$ or $\bar{C}$, and $R$ or $C h_{\mathbb{Q}}$.

Dress also proves a local result which says the following about $C(G)$. Fix a prime $p$, let $\mathcal{H}_{p}$ denote the family of $p$-hyperelementary subgroups and let $C(G)_{p}$ denote the $p$-primary subgroup of $C(G)$. Then

$$
C(G)_{p}=\lim _{\overleftarrow{\mathcal{H}_{p}}} C(H)_{p}=\underset{\overrightarrow{\mathcal{H}_{p}}}{\lim _{\longrightarrow}} C(H)_{p} .
$$

\section{Basic detection}

In $[9,1 . A .4]$ we introduced the category $R G$-Morita, for any commutative ring $R$. The category $\mathbb{Q} G$-Morita is defined as follows. The objects are subgroups, $H \leq G$, and the morphisms from $H_{1}$ to $H_{2}$ are generated by the $H_{2}-H_{1}$ bisets $X$, modulo some relations spelled out in [9, p.249-250]. From [9, 1.A.12(i),p.251], $R(G)$ is a functor on $\mathbb{Q} G$-Morita defined by sending a rational representation $V$ of $H_{1}$ to $\mathbb{Q}[X] \otimes \mathbb{Q} H_{1} V$. Note if $V$ is a permutation module on the $H_{1}$-set $Y$, then $\mathbb{Q}[X] \otimes \mathbb{Q} H_{1} \mathbb{Q}[Y]=\mathbb{Q}\left[X \times_{H_{1}}\right.$ $Y]$ so $P$ is also a functor on $\mathbb{Q} G$-Morita. We proved in [9, 1.A.9,p.251] that the morphisms in $\mathbb{Q} G$-Morita are generated by generalized inductions and restrictions corresponding to homomorphisms $f: G_{1} \rightarrow G_{2}$ which are either injections (subgroups) or surjections (quotient groups).

Theorem 2.1 ([9], 1.A.11, p. 251): The sum of the generalized restriction maps,

$$
C(G) \rightarrow \bigoplus_{B \in \mathcal{B}_{G}} C(B)
$$


is a split injection where $\mathcal{B}_{G}$ denotes the set of basic subquotients of $G$. The sum of the generalized induction maps is a split surjection.

When $G$ is hyperelementary, Theorem 2.1 has a more precise version which will imply the corresponding special case of Berz's theorem. To describe this result, first recall some results from [9]. For each irreducible rational representation $\phi$ there exists a basic subquotient $B$, so that $\phi$ is the generalized induction of $\rho_{B}$ with additional control on the induction. Corresponding to $\phi$ there is an idempotent $e_{\phi}$ in $\mathbb{Q} G$-Morita. This idempotent has the property that if $V$ is any rational representation of $G$, then $e_{\phi} \cdot V=b \phi$ where $\phi$ occurs $b$ times in $V$. If $V$ is a virtual permutation representation so is $e_{\phi} \cdot V$, since $\mathbb{Q} G$-Morita acts on $P(G)$. It follows from this observation that $\alpha_{\phi} \phi \in P(G)$ for $G$ hyperelementary. It is clear that $\alpha_{\phi}$ divides $\alpha_{\rho_{B}}$ since the virtual permutation $\alpha_{\rho_{B}} \rho_{B}$, induced up to $G$, is just $\alpha_{\rho_{B}} \phi$. On the other hand, if $\alpha_{\phi} \phi$ is restricted to $B$ and then hit with $e_{\rho_{B}}$, one gets a virtual permutation representation which is $\alpha_{\phi} \phi$. Hence $\alpha_{\phi}=\alpha_{\rho_{B}}$ and we have shown

Proposition 2.2: For $G$ hyperelementary, $P(G)=\bigoplus_{\phi} \alpha_{\rho_{B}} \mathbb{Z}$ and $\alpha_{\phi}=$ $\alpha_{\rho_{B}}$.

In $\S 4$ we will say more about the $\alpha_{\rho_{B}}$. In particular, for every $p$-hyperelementary group all the $\alpha_{\phi}$ are powers of $p$.

\section{The proof of Theorem A}

To fix some notation for the proof, let $L(G)$ denote the set of all rational representations $\chi$ of $G$ such that $\left\langle\chi, \operatorname{Ind}^{G}\left(\rho_{B}\right)\right\rangle$ is divisible by $a_{\rho_{B}}$ for all basic subquotients $B$ of $G$. Clearly, $L(G)$ is a subgroup of $R(G)$. The goal is to prove $L(G)=P(G)$.

Frobenius reciprocity holds even for generalized restrictions and inductions so

$$
\left\langle\chi, \operatorname{Ind}^{G}\left(\rho_{B}\right)\right\rangle=\left\langle\operatorname{Res}_{B}(\chi), \rho_{B}\right\rangle .
$$

Since virtual permutation representations are also preserved by generalized induction and generalized restriction, it is clear that Frobenius reciprocity implies $P(G) \subseteq L(G)$.

If $G$ is $p$-hyperelementary, the Berz lattice for $G$ equals $\bigoplus_{\phi} \alpha_{\rho_{B}} \mathbb{Z}$. From this it follows that $L(G) \subseteq \bigoplus_{\phi} \alpha_{\rho_{B}} \mathbb{Z}$. Proposition 2.2 now implies $P(G)=L(G)$.

Next note that if $H \leq G$, we have $\operatorname{Res}_{H}(L(G)) \subseteq L(H)$. The proof concludes by induction on the order of $G$. The result is trivial for the trivial group. Assume that $P(H)=L(H)$ for all proper subgroups of $G$. Since $L$ always has restrictions and since it is equal to a Mackey functor on proper 
subgroups, it also has inductions. Hence $L$ is a Mackey functor for the category of finite subgroups of $G$. Since $P(H)=L(H)$ on all hyperelementary subgroups of $G$, proper or not, it follows from Proposition 1.2 that $P(G)=L(G)$.

\section{Basic $p$-hyperelementary groups}

A $p$-hyperelementary group is any group which can be written as an extension, $C \triangleleft G \rightarrow P$ where $C$ is a cyclic group of order prime to $p$ and $P$ is a $p$-group. There is an action map $\psi: P \rightarrow \operatorname{Aut}(C)$. Any such extension is split. The notation follows [9], Sect. 3.A. From [9, 3.A.6,p.272], $G$ is basic if and only if

(1) $p$ is odd and the kernel of $\psi$ is cyclic;

(2) $p=2$ and the kernel of $\psi$ is cyclic, dihedral, semi-dihedral or quaternion and if the kernel is $D(8)$ the conjugation homomorphism $P \rightarrow \operatorname{Out}(D(8))$ is onto.

This includes the theorem of Roquette [15] identifying the basic $p$ groups as cyclic if $p$ is odd and cyclic, quaternionic, semi-dihedral and dihedral of order at least 16 if $p=2$.

An $F$-group is a group with a cyclic normal subgroup $A \triangleleft F \rightarrow$ $F / A$ where the action map $\psi: F / A \rightarrow A u t(A)$ is injective. Each basic $p$ hyperelementary group, $C \triangleleft G \rightarrow P$, has a maximal order cyclic subgroup $A_{p} \leq \operatorname{ker} \psi$ which is normal in $P$. Let $A=A_{p} \times C$. The extension $A \triangleleft$ $G \rightarrow G / A$ displays $G$ as an $F$-group with $G / A$ an abelian $p$-group. This extension is classified by an element $\kappa_{G} \in H^{2}(G / A ; A)$.

From [9, 2.11,p.267], a basic group has a unique faithful rational representation $\rho_{G}$. It is the only irreducible rational representation of $G$ which is faithful when restricted to $A$, and $\operatorname{Res}_{A}\left(\rho_{G}\right)=m_{\rho_{G}} \rho_{A}$. Moreover $\operatorname{Ind}^{G}\left(\rho_{A}\right)$ $=\frac{|G / A|}{m_{\rho_{G}}} \cdot \rho_{G}$.

We can describe the Schur index $m_{\rho_{G}}$ as follows. Let $\Gamma$ denote the Galois group of $\mathbb{Q}\left(\zeta_{|A|}\right)$ over $\mathbb{Q}$. Embedding $A$ as a subgroup of the roots of unity in $\mathbb{Q}\left(\zeta_{|A|}\right)$ determines an isomorphism of $\Gamma$ with $A u t(A)$. Use the map $G / A \rightarrow A u t(A)$ to identify $G / A$ as a subgroup of $\Gamma$. The center field $\mathbb{Q}\left(\rho_{G}\right)$ is just the field corresponding to $G / A$ under the Galois correspondence. There is an induced map

$$
H^{2}(G / A ; A) \rightarrow H^{2}\left(\operatorname{Gal}\left(\mathbb{Q}\left(\zeta_{|A|}\right) / \mathbb{Q}\left(\rho_{G}\right)\right) ; \mathbb{Q}\left(\zeta_{|A|}\right)^{*}\right) \stackrel{j}{\rightarrow} \operatorname{Br}\left(\mathbb{Q}\left(\rho_{G}\right)\right)
$$

where $G / A=\operatorname{Gal}\left(\mathbb{Q}\left(\zeta_{|A|}\right) / \mathbb{Q}\left(\rho_{G}\right)\right), \operatorname{Br}\left(\mathbb{Q}\left(\rho_{G}\right)\right)$ is the Brauer group of the field $\mathbb{Q}\left(\rho_{G}\right)$, and the map labeled $j$ injects its domain onto the set of division algebras with center $\mathbb{Q}\left(\rho_{G}\right)$ which split over $\mathbb{Q}\left(\zeta_{|A|}\right)$. The class $\kappa_{G}$ 
is mapped to the class of the simple factor of $\mathbb{Q} G$ determined by $\rho_{G}$ (see [7, p.193]). The order of this class is $m_{\rho_{G}}$, [13, Thm.32.19,p.280].

We introduce notation to deal with $m_{\rho_{G}}$ locally. By the Benard-Schacher Theorem, [3, 74.20,p.746], the order of the image of $\kappa_{G}$ in one of these local Brauer groups is the same for each prime $\mathfrak{q}$ lying over a fixed prime $q$ of $\mathbb{Q}$, so let $m_{q}\left(\rho_{G}\right)$ denote this common order. At the infinite primes, the same result holds, so let $m_{\mathbb{R}}\left(\rho_{G}\right)$ denote its order. Recall $m_{\mathbb{R}}\left(\rho_{G}\right)$ is either 1 or 2 and it is a result of Frobenius and Schur that it is 2 if and only if $\Sigma_{g \in G} \rho_{G}\left(g^{2}\right)<0$. If $q$ does not divide the order of the group $G$, $m_{q}\left(\rho_{G}\right)=1,\left[3,74.11\right.$, p.740]. Finally, recall that $m_{\rho_{G}}$ is the least common multiple of all the local Schur indices [3, 74.11,p.740]. In our case, $m_{\rho_{G}}$ is a power of $p$ and hence so are all the local Schur indices so the $1 \mathrm{~cm}$ becomes a max. Let $q_{1}, \cdots, q_{r}$ denote the distinct primes dividing $|G|$. Then $m_{\rho_{G}}=\max \left(m_{\mathbb{R}}\left(\rho_{G}\right), m_{q_{1}}\left(\rho_{G}\right), \cdots, m_{q_{r}}\left(\rho_{G}\right)\right)$.

In general it is not easy to compute local Schur indices, but in the discussion below we carry this out in some special cases. We will use the notation $L=\mathbb{Q}\left(\zeta_{|A|}\right)$ and $K=\mathbb{Q}\left(\rho_{G}\right)$. Fix a prime $q \in \mathbb{Q}$, and primes $\mathfrak{q}$ in $K$ and $\mathfrak{Q}$ in $L$ with $\mathfrak{Q} \subset \mathfrak{q} \subset(q)$. Let $\bar{L}_{\mathfrak{Q}}$ denote the residue field of $L_{\mathfrak{Q}}$.

Let $\Gamma_{\mathfrak{Q}}$ denote the decomposition group of $\mathfrak{Q}$ over $\mathbb{Q}$ and let $G_{\mathfrak{Q}}=$ $\Gamma_{\mathfrak{Q}} \cap G / A \leq G / A$ denote the decomposition group of $\mathfrak{Q}$ over $K$. Then the image of $\kappa_{G}$ under the composition

$$
H^{2}(G / A ; A) \stackrel{i^{*}}{\rightarrow} H^{2}\left(G_{\mathfrak{Q}} ; A\right) \stackrel{\mathfrak{s}}{\rightarrow} H^{2}\left(G_{\mathfrak{Q}} ; L_{\mathfrak{Q}}^{*}\right) \stackrel{j_{\mathfrak{Q}}}{\longrightarrow} B r\left(K_{\mathfrak{q}}\right)
$$

determines the image of the local division algebra in its Brauer group. Here $i^{*}$ is the map induced by the inclusion $G_{\mathfrak{Q}} \leq G / A, G_{\mathfrak{Q}}=\operatorname{Gal}\left(L_{\mathfrak{Q}} / K_{\mathfrak{q}}\right)$, and $j_{\mathfrak{Q}}$ is the injection into the Brauer group.

The Galois group $\Gamma_{\mathfrak{Q}}$ maps onto the Galois group of $\bar{L}_{\mathfrak{Q}}$ over $\mathbf{F}_{q}$. The first inertia group is the kernel, denoted $\Gamma_{\mathfrak{Q} 0}$. Let $G_{\mathfrak{2} 0}=\Gamma_{\mathfrak{Q} 0} \cap G / A$ : it is the first inertia group of $\mathfrak{Q}$ over $K$.

There is an exact sequence of $\Gamma_{\mathfrak{Q}}$ modules

$$
0 \rightarrow U \rightarrow L_{\mathfrak{Q}}^{*} \rightarrow \mathbb{Z} \rightarrow 0
$$

where $\mathbb{Z}$ is a trivial $\Gamma_{\mathfrak{Q}}$ module and $U$ is the group of units in the ring of integers of $L_{\mathfrak{Q}}$. It follows that

$$
0=H^{1}\left(G_{\mathfrak{Q}} ; \mathbb{Z}\right) \rightarrow H^{2}\left(G_{\mathfrak{Q}} ; U\right) \rightarrow H^{2}\left(G_{\mathfrak{Q}} ; L_{\mathfrak{Q}}^{*}\right)
$$

is exact and so $\mathfrak{s}$ factors through a map $H^{2}\left(G_{\mathfrak{Q}} ; A\right) \stackrel{\mathfrak{s}^{\prime}}{\rightarrow} H^{2}\left(\Gamma_{\mathfrak{Q}} ; U\right)$. This map can be analyzed by means of the exact sequences

$$
\begin{aligned}
0 & \rightarrow A^{1} \rightarrow A \\
\downarrow & \rightarrow \bar{A} \rightarrow 0 \\
\downarrow & \downarrow \\
0 & \rightarrow U^{1} \rightarrow U
\end{aligned}
$$


where the vertical maps are injections. Recall that there is a Hausdorff filtration on $U^{1}$ so that the associated graded is a vector space over $\bar{L}_{\mathfrak{Q}}$.

From this discussion, we can recover the well-known result on the Schur indices for the quaternion groups:

indices are 1;

$$
m_{\mathbb{R}}\left(\rho_{Q_{8}}\right)=2, m_{2}\left(\rho_{Q_{8}}\right)=2 \text {, and the remaining local Schur }
$$

$r \geq 4$.

$$
m_{\mathbb{R}}\left(\rho_{Q_{2^{r}}}\right)=2 \text {, and all the local Schur indices are } 1 \text { if }
$$

We now describe two further situations where the calculation of the local Schur index is relatively easy.

Case 1. The prime $q$ does not divide $p$. Then

$$
H^{1}\left(G_{\mathfrak{Q}} ; U^{1}\right)=H^{2}\left(G_{\mathfrak{Q}} ; U^{1}\right)=0 .
$$

This follows from [18, Lemma 3,p.185], since $G_{\mathfrak{Q}}$ is a $p$ group and the associated graded is a $\mathbb{Z} / q$ vector space. Hence $H^{2}\left(G_{\mathfrak{Q}} ; U\right) \rightarrow H^{2}\left(G_{\mathfrak{Q}} ; \bar{L}_{\mathfrak{Q}}^{*}\right)$ is an isomorphism. The grading on $U^{1}$ also shows that $A_{p} \rightarrow \bar{A}_{p}$ is an isomorphism and hence $H^{2}\left(G_{\mathfrak{Q}} ; A\right) \rightarrow H^{2}\left(G_{\mathfrak{Q}} ; \bar{A}\right)$ is an isomorphism. Up to these isomorphisms, it suffices to determine the map

$$
H^{2}\left(G_{\mathfrak{Q}} ; \bar{A}\right) \rightarrow H^{2}\left(G_{\mathfrak{2}} ; \bar{L}_{\mathfrak{Q}}^{*}\right) .
$$

Case 2. The group $G_{\mathfrak{Q} 0}=\{e\}$. Equivalently, $\mathfrak{q}$ is unramified over $K$. Then $G_{\mathfrak{Q}}$ is also the Galois group of $\bar{L}_{\mathfrak{Q}}$ over $\bar{K}_{\mathfrak{q}}$. Again $H^{1}\left(G_{\mathfrak{Q}} ; U^{1}\right)=H^{2}\left(G_{\mathfrak{Q}} ; U^{1}\right)=0$ [18, Lemma 2,p.185] and by [18, Ex. a,p.162] we have $H^{2}\left(G_{\mathfrak{Q}} ; \bar{L}_{\mathfrak{Q}}^{*}\right)=0$. Hence $H^{2}\left(G_{, 2} ; U\right)=0$ so the $q$-local Schur index is 0 .

Recall for use below the following fact from algebraic topology.

Lemma 4.1: If $\mathbb{Z} / p^{r}$ acts trivially on cyclic groups $\mathbb{Z} / p^{s}$ and $\mathbb{Z} / p^{t}$ and if $s \leq t$ then the map induced by the inclusion $i: \mathbb{Z} / p^{s} \hookrightarrow \mathbb{Z} / p^{t}$

$$
i_{*}: H^{2}\left(\mathbb{Z} / p^{r} ; \mathbb{Z} / p^{s}\right) \rightarrow H^{2}\left(\mathbb{Z} / p^{r} ; \mathbb{Z} / p^{t}\right)
$$

is the map between cyclic groups which sends a generator of the first to $p^{t-s}$ times a generator of the second.

Proposition 4.2: Let $G$ be a basic p-hyperelementary group. If $G / A$ acts trivially on $A_{p}$, then $m_{p}\left(\rho_{G}\right)=1$. Suppose, in addition, that $|A|=p^{s} \cdot q$ for some prime q, and that $s \leq t$ where $p^{t}$ is the full $p$-power divisor of $q-1$. If $G / A$ has order $p^{r}$, then $m_{q}\left(\rho_{G}\right)$ equals the order of $i_{*}\left(\kappa_{G}\right)$ under the map from Lemma 4.1. Two extreme cases are: if $s=t$, then $m_{q}\left(\rho_{G}\right)$ equals the order of $\kappa_{G}$; if $r+s \leq t$ then $m_{q}\left(\rho_{G}\right)=1$. 
Proof: Since $A=A_{p} \times C, L=\mathbb{Q}\left(\zeta_{p^{s}}, \zeta_{|C|}\right)$ with $\mathbb{Q}\left(\zeta_{p^{s}}\right) \cap \mathbb{Q}\left(\zeta_{|C|}\right)=\mathbb{Q}$. Since $G / A$ acts trivially on $A_{p}, G / A \leq \operatorname{Gal}\left(L / \mathbb{Q}\left(\zeta_{p^{s}}\right)\right)$. To apply Case 2 to prove the first assertion, note that all the $p$-power roots of unity in $L$ are contained in $K$, so the extension $L_{\mathfrak{Q}} / K_{\mathfrak{q}}$ is unramified. Case 2 shows $m_{p}\left(\rho_{G}\right)=1$. Case 1 can be applied to prove the remaining assertions. Here $\zeta_{|C|}=\zeta_{q}$ and $K=\mathbb{Q}\left(\zeta_{q}\right)^{G / A}\left(\zeta_{p^{s}}\right)$. Since $q \equiv 1\left(\bmod p^{s}\right)$, the decomposition group $\Gamma_{\mathfrak{Q}}=\operatorname{Gal}\left(L / \mathbb{Q}\left(\zeta_{p^{s}}\right)\right)$ and the residue class field $\bar{L}_{\mathfrak{Q}}=\mathbf{F}_{q}$. It follows that $G_{\mathfrak{Q}}=G / A$, that $\left(\bar{L}_{\mathfrak{Q}}^{*}\right)_{p} \cong \mathbb{Z} / p^{t}$, and that the action of $G_{\mathfrak{Q}}$ on $\bar{L}_{\mathfrak{Q}}^{*}$ is trivial. Hence the map of $A_{p} \rightarrow\left(\bar{L}_{\mathfrak{Q}}^{*}\right)_{p}$ is just an injection $\mathbb{Z} / p^{s} \rightarrow \mathbb{Z} / p^{t}$. Since $G$ is basic, $G_{\mathfrak{Q}}=G / A=\mathbb{Z} / p^{r}$ for some $r \leq t$, and we must compute the map $i_{*}$ from Lemma 4.1. If $s=t$, the map $i_{*}$ is an isomorphism so $m_{q}\left(\rho_{G}\right)$ is the order of $\kappa_{G}$. If $r+s \leq t$ the map $i_{*}$ is zero, so $m_{q}\left(\rho_{G}\right)=1$.

\section{Calculations for basic $p$-hyperelementary groups}

The goal of this section is to come as close as we can to computing $\alpha_{\rho_{G}}$ for basic $p$-hyperelementary groups. First we prove a general lemma.

Lemma 5.1: Let $U \triangleleft G$ be a normal subgroup. Let $\lambda$ be a representation on $U$ with $\phi=\operatorname{Ind}^{G}(\lambda)$. Let $H$ be any subgroup of $G$, let $L=H \cap U$ and let $\bar{H}=H / L$. Then

$$
\left\langle\phi, \operatorname{Ind}^{G}\left(1_{H}\right)\right\rangle=\frac{1}{|\bar{H}|} \sum_{g \in U \backslash G}\left\langle\operatorname{Res}_{L^{g}}(\lambda), 1_{L^{g}}\right\rangle .
$$

Proof: Consider

$$
\begin{aligned}
\mathbf{S} & :=\sum_{g \in U \backslash G}\left\langle\lambda, \operatorname{Ind}^{U}\left(1_{H^{g} \cap U}\right)\right\rangle \\
& =\sum_{g \in U \backslash G / H}\left(\sum_{h \in H /\left(H^{g} \cap U\right)}\left\langle\lambda, \operatorname{Ind}^{U}\left(1_{H^{g h} \cap U}\right)\right\rangle\right) \\
& =\sum_{g \in U \backslash G / H} \frac{|H|}{\left|H^{g} \cap U\right|}\left\langle\lambda, \operatorname{Ind}^{U}\left(1_{H^{g} \cap U}\right)\right\rangle .
\end{aligned}
$$

To see the second equality, note $H^{g h}=g h H h^{-1} g^{-1}=H^{g}$. The conjugation by $h$ does not change the character $1_{H}$, so all the terms in the second sum are seen to be equal. Note $H^{g} \cap U=\left(H \cap U^{g^{-1}}\right)^{g}=(H \cap U)^{g}=L^{g}$ since $U \triangleleft G$. Hence

$\mathbf{S}=|H / L| \cdot \sum_{g \in U \backslash G / H}\left\langle\lambda, \operatorname{Ind}^{U}\left(1_{H^{g} \cap U}\right)\right\rangle=|H / L| \cdot\left\langle\lambda, \operatorname{Res}_{U}\left(\operatorname{Ind}^{G}\left(1_{H}\right)\right)\right\rangle$ 
where the last equality is just the Mackey double coset formula applied to the composite $\operatorname{Res}_{U}\left(\operatorname{Ind}^{G}\left(1_{H}\right)\right)$. But

$$
\left\langle\phi, \operatorname{Ind}^{G}\left(1_{H}\right)\right\rangle=\left\langle\operatorname{Ind}^{G}(\lambda), \operatorname{Ind}^{G}\left(1_{H}\right)\right\rangle=\left\langle\lambda, \operatorname{Res}_{U}\left(\operatorname{Ind}^{G}\left(1_{H}\right)\right)\right\rangle
$$

by Frobenius, so

$$
|\bar{H}| \cdot\left\langle\phi, \operatorname{Ind}^{G}\left(1_{H}\right)\right\rangle=\mathbf{S}=\sum_{g \in U \backslash G}\left\langle\operatorname{Res}_{L^{g}}(\lambda), 1_{L^{g}}\right\rangle
$$

by Frobenius reciprocity applied to $\left\langle\lambda, \operatorname{Ind}^{U}\left(1_{H^{g} \cap U}\right)\right\rangle$.

Proposition 5.2: For $G$ basic p-hyperelementary, $a_{\rho_{G}}$ is the gcd of the numbers $\frac{\rho_{A}(1) m_{\rho_{G}}}{|H|}$ as $H$ runs over all subgroups of $G$ with $H \cap A=\{e\}$ : $\alpha_{\rho_{G}}$ is the gcd of the numbers $\frac{|G|}{|A| \cdot|H| \cdot m_{\rho_{G}}}$ running over the same $H$.

Proof: Apply Lemma 5.1 to $U=A$ with $\lambda$ an irreducible complex constituent of $\rho_{A}$. Since $\lambda$ is faithful, the inner products on the right are 0 unless $H^{g} \cap A=L^{g}=\{e\}$ when they are all 1 . Next recall that $\rho_{A}$ is just a sum of Galois conjugates of $\lambda$ and recall that $\operatorname{Ind}^{G}\left(\rho_{A}\right)=\frac{|G / A|}{m_{\rho_{G}}} \cdot \rho_{G}$. Since $\left\langle\rho_{G}, \rho_{G}\right\rangle=\frac{m_{\rho_{g}}^{2} \cdot \rho_{A}(1)}{|G / A|}$, the result follows.

Lemma 5.3: $C(G)$ is a p-torsion group if $G$ is $p$-hyperelementary.

Proof: Since subquotients of $p$-hyperelementary groups are again $p$-hyperelementary the result follows from Proposition 5.2 and Proposition 2.2.

Since the gcd of a set of powers of $p$ is just the minimum, the following is an immediate consequence of Proposition 5.2.

Proposition 5.4: Given a hyperelementary basic group $G$, there exist subgroups $H$ such that $H$ has maximal order subject to the condition $H \cap A=$ $\{e\}$. For any such $H, \alpha_{\rho_{G}}=\frac{|G|}{|A| \cdot|H| \cdot m_{\rho_{G}}}$ and $a_{\rho_{G}}=\frac{\varphi(|A|) m_{\rho_{G}}}{|H|}$ where $\varphi(n)$ is Euler's phi function of the integer $n$.

Proposition 5.5: If $G$ is basic and $A \triangleleft G \rightarrow G / A$ is split, $m_{\rho_{G}}=1$ and $\alpha_{\rho_{G}}=1$.

Proof: Take $H=G / A$ in Proposition 5.4 and recall $\alpha_{\rho_{G}}$ is an integer.

Corollary 5.6: Let $G$ be a finite group with elementary abelian p-Sylow subgroup. Then $C(G)_{p}=0$. 
Proof: By (2.1) and (2.2) it is enough to compute $\alpha_{\rho_{G}}$ for basic subquotients of $G$. Now apply (5.3) and Proposition 5.5.

Sometimes a basic $p$-hyperelementary group can be decomposed as $G=$ $E \times G^{\prime}$ where the order of $E$ is prime to $p$. Note that $G^{\prime}$ is automatically basic and that the basic representation $\rho_{G}$ is just $\rho_{E} \otimes \rho_{G^{\prime}}$. For a given $G$ there is a unique maximal direct factor $E$ of order prime to $p$.

Lemma 5.7: Let $G=E \times G^{\prime}$ be a basic p-hyperelementary group, with the order of $E$ prime to $p$. Then $m_{\rho_{G}} \cdot \alpha_{\rho_{G}}=m_{\rho_{G^{\prime}}} \cdot \alpha_{\rho_{G^{\prime}}}$. The Schur index $m_{\rho_{G}}=m_{\rho_{G^{\prime}}}$ if $E=\mathbb{Z} / 2$ or $\{e\}$. Otherwise $m_{\mathbb{R}}\left(\rho_{G}\right)=1, m_{q}\left(\rho_{G}\right)=1$ if $\left(q,\left|G^{\prime}\right|\right)=1$, and $m_{q}\left(\rho_{G}\right)=m_{q}\left(\rho_{G^{\prime}}\right) /\left(f_{q}(E), m_{q}\left(\rho_{G^{\prime}}\right)\right)$ where $f_{q}(E)$ is the order of $q \in(\mathbb{Z} /|E|)^{\times}$if $q$ divides $\left|G^{\prime}\right|$.

Proof: The first assertion follows from Proposition 5.4: the quantity $m_{\rho_{G}}$. $\alpha_{\rho_{G}}$ for a basic $p$-hyperelementary group depends only on the $p$-Sylow subgroup. The calculation of the change in Schur index is easy to describe at the level of simple factors of the group rings: the center field for the factor for $\rho_{G^{\prime}}$ in $\mathbb{Q}\left[G^{\prime}\right]$ is just extended by tensoring with $\mathbb{Q}\left(\zeta_{|E|}\right)=\mathbb{Q}\left(\rho_{E}\right)$. Since the order of $E$ is prime to the order of $G^{\prime}$, the new center field is just $\mathbb{Q}\left(\rho_{G}\right)$ and we compute the local invariants of this extended factor.

The result is immediate for $|E| \leq 2$, so assume $|E|>2$. Since the field $\mathbb{Q}\left(\rho_{G}\right)$ is totally imaginary, $m_{\mathbb{R}}\left(\rho_{G}\right)=1$. If $m_{q}\left(\rho_{G^{\prime}}\right)=1$ then under the extension it remains split: in particular if $\left(q,\left|G^{\prime}\right|\right)=1, m_{q}\left(\rho_{G}\right)=1$.

We now turn to the primes dividing $\left|G^{\prime}\right|$. Since the order of $E$ is prime to the order of $G^{\prime}$, we can reduce to the cyclotomic case as follows. Let $\mathfrak{Q}$ be a prime of $\mathbb{Q}\left(\rho_{G^{\prime}}\right)$ lying over the prime $q$ of $Q$. If $q$ splits in $\mathbb{Q}\left(\rho_{E}\right)$ into $\bar{q}_{1}, \cdots, \bar{q}_{g}$, then $\mathfrak{Q}$ splits in $\mathbb{Q}\left(\rho_{G}\right)$ into $\overline{\mathfrak{Q}}_{1}, \cdots, \overline{\mathfrak{Q}}_{g}$. The main issue here is the degree of the extension $\widehat{\mathbb{Q}}\left(\rho_{G}\right)_{\overline{\mathfrak{Q}}_{i}}$ over $\widehat{\mathbb{Q}}\left(\rho_{G^{\prime}}\right)_{\mathfrak{Q}_{i}}$. By Galois theory, this equals the degree of $\widehat{\mathbb{Q}}\left(\rho_{E}\right)_{\bar{q}_{i}}$ over $\widehat{\mathbb{Q}}_{q_{i}}$.

The classical theory says that $q_{i}$ is unramified in $\mathbb{Q}\left(\rho_{E}\right)$ and that the degree of the residue field extension is the order of $q_{i}$ in $(\mathbb{Z} /|E|)^{\times}$, denoted $f_{q_{i}}(E)$ and therefore the degree of $\widehat{\mathbb{Q}}\left(\rho_{E}\right)_{\bar{q}_{i}}$ over $\widehat{\mathbb{Q}}_{q_{i}}$ is just $f_{q_{i}}(E)$.

Each local Brauer group at a finite place is a $\mathbb{Q} / \mathbb{Z}$. The map on the sum of the local Brauer groups is a diagonal map into $g$ copies of $\mathbb{Q} / \mathbb{Z}$ corresponding to the splitting of the prime $\mathfrak{Q}$ preceded by multiplication by the degree of the local field extension $f_{q_{i}}(E)$ (see [18, Prop.7,p.193]).

As an application of Lemma 5.7, we consider the product of cyclic groups with quaternion groups. The prime 2 has order 2 in $(\mathbb{Z} / 3)^{\times}$. Hence $m_{2}\left(\rho_{\mathbb{Z} / 3 \times Q_{8}}\right)=1$ which is one explanation of Serre's example. But 2 has order 3 in $(\mathbb{Z} / 7)^{\times}$so $m_{2}\left(\rho_{\mathbb{Z} / 7 \times Q_{8}}\right)=2$ and for $\mathbb{Z} / 7 \times Q_{8}$ all rational repre- 
sentations are permutation representations. For any odd prime $p$ and $r \geq 4$ we have $m_{2}\left(\rho_{\mathbb{Z} / p \times Q_{2^{r}}}\right)=1$.

Lemma 5.7 gives a partial converse to Proposition 5.5.

Corollary 5.8: Let $G$ be a $p$-hyperelementary basic group. If $\kappa_{G} \neq 0$ then there exists a cyclic group $E$ of order prime to $p$ such that p divides $\alpha_{\rho_{E \times G}}$.

Proof: Since $\kappa_{G} \neq 0, p$ divides $m_{\rho_{G}} \cdot \alpha_{\rho_{G}}$. Let $p^{t}$ be the exact power of $p$ dividing $m_{\rho_{G}}$ and let $\ell_{1}, \cdots, \ell_{r}$ be the distinct primes dividing the order of $G$

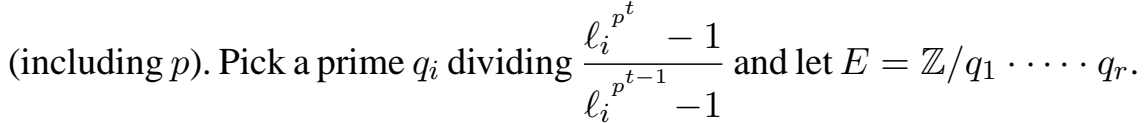
Use Lemma 5.7 to see $m_{\rho_{E \times G}}=1$ so $p$ divides $\alpha_{\rho_{E \times G}}$.

Note that any prime divisor $q$ of $\frac{\ell^{p^{t}}-1}{\ell^{p^{t-1}}-1}$ will have $p^{t}$ as the order of $\ell$ in $(\mathbb{Z} / q)^{\times}$.

\section{Calculations for a $p$-group}

If $G$ is a basic $p$-group, then $G / A$ is trivial or $\mathbb{Z} / 2$. The group $G / A$ is trivial except when $G$ is dihedral, semi-dihedral or quaternion. The extension $A \triangleleft$ $G \rightarrow G / A$ is split if $G$ is dihedral or semi-dihedral and is non-split if $G$ is quaternion. In this last case, the Schur index is 2 . Therefore in all cases, $\alpha_{\rho_{G}}=1$.

Theorem 6.1 (Ritter-Segal): $C(G)=0$ for $G$ a $p$-group.

Proof: The result follows from Proposition 2.2 and the calculation that $\alpha_{\rho_{G}}=1$ for basic $p$-groups $G$. This line of argument was attributed to W. Feit in the introduction to [16].

For a basic $p$-group, $\bar{C}(G)=0$ if $G$ is cyclic, dihedral or semidihedral, and $\bar{C}\left(Q_{2^{r}}\right)=\mathbb{Z} / 2$. This follows from (0.2) and our previous remarks about Schur indices.

Proposition 6.2: For any $p-$ group, $p$ odd, $\bar{C}(G)=0$. For any finite 2group $G$ there exists a set $\mathcal{B}$ of quaternionic subquotients so that both the generalized induction and the generalized restriction induce an isomorphism between $\bar{C}(G)$ and $\underset{Q_{2^{r} \in \mathcal{B}}}{\bigoplus} \bar{C}\left(Q_{2^{r}}\right)$.

Proof: It follows in general from [9, 4.A.8,p.283] that there is a set of basic subquotients $\mathcal{B}^{\prime}$ so that for any functor $F$ on $\mathbb{Q} G$-Morita, $F(G)$ and $\bigoplus_{B \in \mathcal{B}^{\prime}} e_{\rho_{B}} \cdot F(B)$ are isomorphic via either the generalized induction or the 
generalized restriction. For the case $F=\bar{C}$, only the quaternion subquotients can make a non-zero contribution and the idempotent factor $e_{\rho_{B}} \bar{C}(B)$ is also $\mathbb{Z} / 2$ as one sees by applying the general result to $Q_{2^{r}}$.

Discussion: The defining property of the basic subquotients in $\mathcal{B}^{\prime}$ is that $\rho_{B}$ goes to an irreducible rational representation $\phi$ of $G$ under the generalized induction and that $\phi$ and $\rho_{B}$ have the same center and division algebra (hence the same Schur index). Therefore the number of elements in $\mathcal{B}$ and the orders of the various $Q_{2^{r}}$ 's that occur can be read off if one knows the Wedderburn decomposition of $\mathbb{Q}[G]$. Alternately, an irreducible rational character $\phi$ is of quaternion type if and only if $\sum_{g \in G} \phi\left(g^{2}\right)<0$; if it is of quaternion type, then $\langle\phi, \phi\rangle=4 \cdot 2^{r-3}=2^{r-1}$ determines the corresponding quaternion subquotient group $Q_{2^{r}}$. If one knows the complex characters, look for irreducible characters $\chi$ so that $\sum_{g \in G} \chi\left(g^{2}\right)=-1$. Work out the orbits under Galois conjugacy of these characters. The number of orbits is the cardinality of $\mathcal{B}$ and an orbit with $2^{r-3}$ elements corresponds to a $Q_{2^{r}} \in \mathcal{B}$.

Notation: The isomorphism in Proposition 6.2 introduces a direct sum decomposition on $\bar{C}(G)$ by the orders of the quaternion subquotients. For us it will only be important whether the quaternion group is $Q_{8}$ or not, so let $\bar{C}(G)_{8}$ denote the summand of $\bar{C}(G)$ corresponding to subquotients $Q_{8}$ and let $\bar{C}(G)_{\geq 16}$ denote the summand corresponding to the subquotients of $Q_{16}$ and larger.

\section{Examples of large $C(G)$}

We can construct groups with large torsion in $C(G)$ as follows. Fix a prime $p$ and integers $r, s$ and $t$ with $0<r \leq t$ and $0<s \leq t$. Let $q$ be any prime with $q=1+b \cdot p^{t}$ with $p \nmid b$, and form $G=\mathbb{Z} / q \rtimes \mathbb{Z} / p^{s+r}$. The action is defined by the composition $\mathbb{Z} / p^{s+r} \rightarrow \mathbb{Z} / p^{r} \hookrightarrow A u t(\mathbb{Z} / q)$. Note that $G$ is basic $p$-hyperelementary and that the extension class $\kappa_{G}$ has order $p^{r}$. A computation using Lemma 4.1 and Proposition 4.2 shows that the Schur index $m_{\rho_{G}}=p^{r+s-t}$ if $t<r+s$ and $m_{\rho_{G}}=1$ otherwise. By Proposition 5.4, it follows that $\alpha_{\rho_{G}}=p^{t-s}$ if $t<r+s$ and $\alpha_{\rho_{G}}=p^{r}$ otherwise. Therefore $C(G)$ has a $\mathbb{Z} / p^{r}$ summand coming from the basic representation of $G$ whenever $t \geq r+s$.

Since $C$ is a functor on $\mathbb{Q} G$-Morita, $C\left(G^{\prime} \times G^{\prime \prime}\right)$ contains $C\left(G^{\prime}\right) \oplus C\left(G^{\prime \prime}\right)$ as a summand. We can now construct examples of groups $G$ which contain a given finite abelian group as a summand of $C(G)$. Note further that the groups constructed are all metabelian, so these also provide examples where the Berz lattice has large index in $R(G)$. 


\section{Vanishing results and preliminary calculations}

This next result will enable us to calculate $\bar{C}(G)$ in many cases needed later.

Lemma 8.1: Let $G=G_{0} \times G_{1}$ with $\left(\left|G_{0}\right|,\left|G_{1}\right|\right)=1$. Then

$$
\gamma_{G_{0} \times G_{1}}: \bar{C}\left(G_{0} \times G_{1}\right) \rightarrow \bar{C}\left(G_{0}\right) \otimes C h_{\mathbb{Q}}\left(G_{1}\right) \oplus C h_{\mathbb{Q}}\left(G_{0}\right) \otimes \bar{C}\left(G_{1}\right)
$$

is an isomorphism. Given $G_{0}^{\prime} \leq G_{0}$

$$
\begin{aligned}
& \bar{C}\left(G_{0} \times G_{1}\right) \stackrel{\gamma_{G_{0} \times G_{1}}}{\longrightarrow} \bar{C}\left(G_{0}\right) \otimes C h_{\mathbb{Q}}\left(G_{1}\right) \oplus C h_{\mathbb{Q}}\left(G_{0}\right) \otimes \bar{C}\left(G_{1}\right) \\
& \operatorname{Res}_{G_{0}^{\prime} \times G_{1}} \downarrow \\
& \bar{C}\left(G_{0}^{\prime} \times G_{1}\right) \stackrel{\gamma_{G_{0}^{\prime} \times G_{1}}}{\longrightarrow} \bar{C}\left(G_{0}^{\prime}\right) \otimes C h_{\mathbb{Q}}\left(G_{1}\right) \oplus C h_{\mathbb{Q}}\left(G_{0}^{\prime}\right) \otimes \bar{C}\left(G_{1}\right) \oplus\left(\operatorname{Res}_{G_{0}^{\prime}} \otimes 1\right)
\end{aligned}
$$

commutes.

Proof: The Burnside ring is the free abelian group on cosets, $G$ / $H$ as $H$ runs over the conjugacy classes of subgroups of $G$. For $G$ as in the Lemma, $\left(G_{0} \times\right.$ $\left.G_{1}\right) / H=\left(G_{0} / H_{0}\right) \times\left(G_{1} / H_{1}\right)$, so it follows that $P\left(G_{0} \times G_{1}\right)=P\left(G_{0}\right) \otimes$ $P\left(G_{1}\right)$. Each irreducible complex representation of $G$ is the tensor product of complex representations on $G_{0}$ and $G_{1}$, even without the assumption on the orders. The assumption on the orders guarantees that $C h_{\mathbb{Q}}\left(G_{0} \times G_{1}\right)=$ $C h_{\mathbb{Q}}\left(G_{0}\right) \otimes C h_{\mathbb{Q}}\left(G_{1}\right)$ because the tensor product of two fields of relatively prime orders remains a field. The calculation follows. The commutativity of the square follows from the behavior of the tensor product of characters under restriction.

The next goal is to calculate $C(G)$ when $G$ is a $p$-elementary group: i.e. $G=E \times G_{p}$ where $E$ is cyclic of order prime to $p$ and $G_{p}$ is a $p$-group. Note that $C(E)=0$ for $E$ any cyclic group (see [17, Ex. 13.1(c),p.105]). Since the Schur indices are trivial, $\bar{C}(E)=0$ as well.

Proposition 8.2: If $G$ is a p-elementary group, $C(G)=\bar{C}(G)=0$ if $p$ is odd. If $p=2$ both $C(G)$ and $\bar{C}(G)$ are $\mathbb{Z} / 2$ vector spaces which vanish if $\bar{C}\left(G_{2}\right)=0$.

Proof: Since $\bar{C}(E)=0$, Lemma 8.1 shows $\bar{C}(G)=R(E) \otimes \bar{C}\left(G_{p}\right)$. By Theorem 2.1 and Proposition 6.2, $\bar{C}\left(G_{p}\right)=0$ if $p$ is odd and is a $\mathbb{Z} / 2$ vector space if $p=2$.

We say that a Mackey functor $\mathcal{M}$ is $p$-elementary generated for $G$ provided the sum of induction maps $\bigoplus_{H} \mathcal{M}(H) \rightarrow \mathcal{M}(G)$ is onto where $H$ runs over the $p$-elementary subgroups of $G$. We say that it is $p$-elementary detected for $G$ provided the sum of restriction maps $\mathcal{M}(G) \rightarrow \bigoplus_{H} \mathcal{M}(H)$ is injective. The next result is immediate from Proposition 8.2. 
Corollary 8.3: Let $p$ be a prime. If $p$ is odd and $\bar{C}_{p}$ is p-elementary generated or detected for $G$, then $C(G)_{p}=\bar{C}(G)_{p}=0$. If $\bar{C}_{2}$ is 2-elementary generated or detected for $G$, then $C(G)_{2}$ and $\bar{C}(G)_{2}$ are $\mathbb{Z} / 2$ vector spaces: if in addition $\bar{C}(K)=0$ for $K$ any subgroup of the 2-Sylow subgroup of $G$, then $C(G)_{2}=\bar{C}(G)_{2}=0$.

The $p$-localization of any hyperelementary computable Mackey functor, such as $C$ or $\bar{C}$, will be $p$-elementary computable for $G$ any time all the $p$-hyperelementary subgroups of $G$ are $p$-elementary. This happens in a variety of situations, for example:

1. If $p$ is the largest prime dividing the order of $G$, any $p$-hyperelementary subgroup is $p$-elementary. Theorem $\mathrm{B}$ is an immediate consequence if $p>2$ and follows from Theorem 6.1 if $p=2$.

2. If $p$ is a prime dividing the order of $G$ and $\left(p, \varphi\left(\left|G / G_{p}\right|\right)\right)=1$ any $p$-hyperelementary subgroup is $p$-elementary.

3 . If the $p$-Sylow subgroup of $G$ is normal, any $p$-hyperelementary subgroup of $G$ is $p$-elementary.

\section{Idempotents in the 2-local Burnside ring}

In addition to induction theory, Dress constructed idempotents in the local Burnside ring. In [8, Sect. 6] and [12, Sect. 11] these idempotents are combined with induction theory to do calculations. We only discuss the 2-local case on a group $G_{\text {odd }}$ of odd order. Dress constructs one idempotent $e_{E}$ in the 2-local Burnside ring for each conjugacy class of cyclic subgroups of $G_{\text {odd }}$. One can then split any 2-local Mackey functor using these idempotents, and the main result we want is Oliver's identification of the pieces $[12,11.5, \mathrm{p} .256]$. Let $F$ be a $2-$ local Mackey functor on $G_{\text {odd }}$ which is 2hyperelementary computable. Then for each cyclic subgroup $E$ and each subgroup $K$ of $G_{\text {odd }}$,

$$
\left(e_{E} \cdot F\right)(K)=\left(e_{E} \cdot F\right)(E)^{N_{K}(E)} .
$$

In general Oliver describes the answer as a limit over subgroups of $N_{K}(E)$ of the form $E \leq H \rightarrow P$ where $P$ is a 2-group. Since $K$ has odd order, $E$ is the unique such group and all that remains of the limit is to take the fixed subgroup. Hence we have

Theorem 9.1: Let $F$ be a 2-local Mackey functor on a group of odd order. Let $F$ be 2-hyperelementary computable. Then for any subgroup $K$

$$
F(K)=\bigoplus_{E \in \mathcal{E}_{K}}\left(\left(e_{E} \cdot F\right)(E)\right)^{N_{K}(E)} .
$$


The next task is to calculate the idempotent $e_{E}$. We should really do this in the 2-local Burnside ring, but for a cyclic group the Burnside ring and the rational representation ring are isomorphic, and it will be more convenient to have the answer in terms of representations anyway. To fix some notation, let $E$ be a cyclic group and $d$ a divisor of $|E|$. Define $\lambda_{d}^{E}$ to be the irreducible rational representation obtained by pulling the faithful irreducible rational representation on $\mathbb{Z} / d$ back to $E$ under an epimorphism $E \rightarrow \mathbb{Z} / d$ (there are usually several but the representation is independent of choice).

Lemma 9.2: The idempotent $e_{E}$ for the odd order cyclic group $E$ in the 2-local Burnside ring is

$$
e_{E}=\otimes_{i=1}^{r}\left(\frac{\left(p_{i}-1\right) 1_{E}-\lambda_{p_{i}}^{E}}{p_{i}}\right)
$$

where $|E|=\prod_{i=1}^{r} p_{i}^{s_{i}}$ with $s_{i}>0$ for all $i: e_{\{e\}}=1_{\{e\}}$.

Proof: Provisionally, let $\widehat{e}$ denote the element on the right hand side of our formula. The formula $\lambda_{p}^{E} \otimes \lambda_{p}^{E}=(p-1) 1_{E}+(p-2) \lambda_{p}^{E}$ can be used to show $\widehat{e}$ is an idempotent. If one works in $\mathbb{Z} / 2 \otimes R$ then $\widehat{e}=\otimes_{i=1}^{r} \lambda_{p_{i}}^{E}$ and this is an irreducible rational representation of $E$. Hence $\widehat{e}$ generates a summand of $\mathbb{Z}_{(2)} \otimes R$.

It follows from [8, Prop. 6.17,p.821] that $\left(e_{E} \cdot \mathbb{Z}_{(2)} \otimes R\right)(E) \cong \mathbb{Z}_{(2)} \otimes$ $K_{0}\left(\mathbb{Q}\left(\zeta_{|E|}\right)\right)=\mathbb{Z}_{(2)}$. There is also a less mysterious description of $e_{E}$ (see [8, (6.12),p.819]). If $E^{\prime}$ runs over the maximal proper subgroups of $E$,

$$
0 \rightarrow\left(e_{E} \cdot F\right)(E) \rightarrow F(E) \stackrel{\oplus \operatorname{Res}_{E^{\prime}}}{\longrightarrow} \bigoplus_{E^{\prime}} F\left(E^{\prime}\right)
$$

is split exact for any 2-local Mackey functor including $\mathbb{Z}_{(2)} \otimes R$. One can see $\operatorname{Res}_{E^{\prime}}(\widehat{e})=0$ since one of the elements in the tensor product will vanish. Therefore $\widehat{e}$ is contained in $\left(e_{E} \cdot \mathbb{Z}_{(2)} \otimes R\right)(E)$ and hence must be $e_{E}$. The formula for $e_{\{e\}}$ is obvious.

\section{The calculation of $C(G)$ and $\bar{C}(G)$ when $G_{2} \triangleleft G$}

Let $G / G_{2}=G_{\text {odd }}$. The analysis promised proceeds in several steps. For any group $K$ let $\iota_{K}: C(K) \rightarrow \bar{C}(K)$ denote the inclusion. Define a 2-local Mackey functor on $G_{\text {odd }}$ by $F(K)=C\left(\pi^{-1}(K)\right)$ where $\pi: G \rightarrow G_{\text {odd }}$ is the projection. Define $\bar{F}(K)=\bar{C}\left(\pi^{-1}(K)\right)$. Recall $\pi^{-1}(E)=E \times G_{2}$ whenever $E$ is cyclic.

Recall from the Introduction that a prime $\ell$ is non-split if and only if 2 has odd order in $(\mathbb{Z} / \ell)^{\times}$. Call a cyclic group $E$ non-split if all the primes dividing its order are non-split: otherwise, call it split. 
Choose a basis of $\bar{C}\left(G_{2}\right)$ by irreducible rational-valued class functions: $\chi_{1}, \cdots, \chi_{s}$ corresponding to $Q_{8}$ subquotients and $\eta_{1}, \cdots, \eta_{t}$ corresponding to $Q_{2^{r}}$ subquotients with $r \geq 4$.

Finally, recall the the representations $\lambda_{p_{i}}^{E}$ from Lemma 9.2. The main calculation is

Theorem 10.1: If $E$ is a cyclic group with $|E|$ odd and $>1$, then $\bar{F}(E)=$ $R(E) \otimes \bar{C}\left(G_{2}\right)$ :

$$
\begin{aligned}
& \left(e_{E} \cdot \bar{F}\right)(E)=\bar{C}\left(G_{2}\right) \\
& \left(e_{E} \cdot F\right)(E)= \begin{cases}\bar{C}\left(G_{2}\right) & \text { if } E \text { is split } \\
\bar{C}\left(G_{2}\right)_{\geq 16} & \text { if } E \text { is non-split }\end{cases}
\end{aligned}
$$

A basis for the summand $\left(e_{E} \cdot \bar{F}\right)(E)$ is given by the tensor products $\left(\otimes_{i=1}^{r} \lambda_{p_{i}}^{E}\right) \otimes \chi_{j}$ and $\left(\otimes_{i=1}^{r} \lambda_{p_{i}}^{E}\right) \otimes \eta_{j}$. The map $\iota_{E}$ preserves the idempotent decomposition and restricts to the identity on the $e_{E}$ summand if $E$ is split. If $E$ is non-split the restriction of $\iota_{E}$ to the $e_{E}$ summand is the evident inclusion.

Proof: That $\bar{F}(E)=R(E) \otimes \bar{C}\left(G_{2}\right)$ follows immediately from Lemma 8.1. A basis for it is given by the $\phi_{i} \otimes \chi_{j}$ and $\phi_{i} \otimes \eta_{j}$ where the $\phi_{i}$ run over the irreducible rational representations of $E$.

Since $\bar{C}\left(G_{2}\right)$ is a $\mathbb{Z} / 2$ vector space, we can use $\otimes_{i=1}^{r} \lambda_{p_{i}}^{E}$ for the idempotent: see Lemma 9.2. Hence $\left(e_{E} \cdot \bar{F}\right)(E)$ is as claimed and situated in $\bar{F}(E)$ as claimed.

Recall the isomorphism $S: \bar{C} / C \rightarrow \bigoplus_{\phi} \mathbb{Z} / m_{\phi}$ from (0.2). For our basis elements $\phi=\left(\otimes_{i=1}^{r} \lambda_{p_{i}}^{E}\right) \otimes \chi_{j}$ or $\phi=\left(\otimes_{i=1}^{r} \lambda_{p_{i}}^{E}\right) \otimes \eta_{j}$ the image $S(\phi)$ is the Schur index of $\phi$. From Lemma 5.7 we see that the $\left(\otimes_{i=1}^{r} \lambda_{p_{i}}^{E}\right) \otimes \eta_{j}$ always have Schur index 1, but that each $\left(\otimes_{i=1}^{r} \lambda_{p_{i}}^{E}\right) \otimes \chi_{j}$ has a 2-local Schur index which is 2 if 2 has odd order in $(\mathbb{Z} /|E|)^{\times}$and is 1 otherwise. Therefore the order of 2 in $(\mathbb{Z} /|E|)^{\times}$is odd if and only if all the primes dividing $|E|$ are non-split. We have established the calculation of $\left(e_{E} \cdot F\right)(E)$. The claim about $\iota_{E}$ follows from checking it on the bases just given.

In what follows, let $\mathcal{E}_{G_{\text {odd }}}^{\text {split }}$ denote the congugacy classes of $E$ such that $E \neq\{e\}$ and $E$ is split. Let $\mathcal{E}_{G_{\text {odd }}}^{\text {nonsplit }}$ denote the congugacy classes of $E$ such that $E \neq\{e\}$ and $E$ is non-split.

Corollary 10.2: Suppose the 2-Sylow subgroup $G_{2}$ of $G$ is normal. Then

$$
\begin{aligned}
& \bar{C}(G)_{2} \cong \bigoplus_{E \in \mathcal{E}_{G_{\text {odd }}}} \bar{C}\left(G_{2}\right)^{N_{G_{\text {odd }}}(E)} \\
& C(G)_{2} \cong \bigoplus_{E \in \mathcal{E}_{G_{\text {odd }}^{\text {split }}}} \bar{C}\left(G_{2}\right)^{N_{G_{\text {odd }}}(E)} \oplus \underset{\substack{E \in \mathcal{E}_{G_{\text {odd }}^{\text {nonsplit }}} \\
\bigoplus}}{ }\left(\bar{C}\left(G_{2}\right)_{\geq 16}\right)^{N_{G_{\text {odd }}}(E)}
\end{aligned}
$$


The inclusion $\iota_{G}$ respects the summands.

Proof: Apply Theorem 9.1 to the functors $F$ and $\bar{F}$. Use Theorem 10.1 to evaluate the summands for $|E| 1$. For $E=\{e\}$, use (9.2) and Theorem 6.1 to evaluate the summand $F(\{e\})$.

One can construct examples of groups of odd order permuting the factors in a direct sum of quaternion groups, and in such examples the fixed sets can be proper subspaces. Assuming that $G=G_{2} \times G_{\text {odd }}$, each fixed point set in Corollary 10.2 is the whole subspace. Lemma 8.1 gives the nice description $\bar{C}(G)_{2} \cong \widetilde{R}\left(G_{\text {odd }}\right) \otimes \bar{C}\left(G_{2}\right)$, but it is still difficult to identify the subgroup $C(G)_{2}$ in a functorial way. One solution is to recall the chosen collection of quaternion subquotients, $Q_{2^{r}} \subset \mathcal{B}$.

Corollary 10.3: Under the generalized induction maps,

$$
\begin{gathered}
\bigoplus_{Q_{2^{r} \in \mathcal{B}}} \bar{C}\left(G_{\text {odd }} \times Q_{2^{r}}\right)_{2} \rightarrow \bar{C}(G)_{2} \\
\bigoplus_{Q_{2^{r} \in \mathcal{B}}} C\left(G_{\text {odd }} \times Q_{2^{r}}\right)_{2} \rightarrow C(G)_{2}
\end{gathered}
$$

are isomorphisms. There are similar isomorphisms using the generalized restrictions.

Proof: Using Theorem 9.1, write down a basis for both sides of the isomorphism and observe that by construction the generalized induction map takes each basis element in the domain to a basis element in the range and that every basis element in the range is accounted for under this correspondence.

The same proof works for the generalized restriction maps, but here is a different one. Define a Mackey functor $\mathcal{J}$ on $G_{\text {odd }}$ by $\mathcal{J}(K)=\bigoplus_{Q_{2^{r}} \in \mathcal{B}}$ $C\left(K \times Q_{2^{r}}\right)_{2}$ for any $K \leq G_{\text {odd }}$. Because $G=G_{2} \times G_{\text {odd }}$, the sum of the generalized restriction maps define a natural transformation of Mackey functors, $\beta_{K}: F(K) \rightarrow \mathcal{J}(K)$. Check that $\beta_{E}$ is an isomorphism for cyclic subgroups $E$ and use Theorem 9.1 to finish.

Another solution to describing our calculation succinctly is to assume a bit more. Suppose that there exists a normal subgroup $G_{s} \triangleleft G_{\text {odd }}$ such that only split primes divide the order of $G_{s}$ and only non-split primes divide the order of $G_{\text {odd }} / G_{s}=G_{n s}$. In this case say $G_{\text {odd }}$ has a normal split subgroup.

Corollary 10.4: If there is a normal split subgroup in $G_{\text {odd }}$ and if $G=$ $G_{2} \times G_{\text {odd }}$, then

$$
C(G)_{2} \cong V \otimes \bar{C}\left(G_{2}\right)_{8} \oplus \widetilde{R}\left(G_{\text {odd }}\right) \otimes \bar{C}\left(G_{2}\right)_{\geq 16}
$$

where $V$ denotes the kernel of the restriction map $R\left(G_{\text {odd }}\right) \rightarrow R\left(G_{n s}\right)$. 
Proof: Observe that non-split cyclic subgroups of $G_{\text {odd }}$ correspond to nontrivial cyclic subgroups of $G_{n s}$ under the projection.

Note that the formula in Theorem $\mathrm{C}$ now follows since all the hypotheses of Corollary 10.4 are satisfied and the kernel of $R\left(G_{\text {odd }}\right) \rightarrow R\left(G_{n s}\right)$ can be identified with $\widetilde{R}\left(G_{s}\right) \otimes R\left(G_{n s}\right)$.

The formula in Theorem $C^{\prime}$ follows from (10.2): all the quaternion representations $\xi$ of $G_{2}$ have $m_{\mathbb{R}}(\xi)=2$ and $Q_{8}$ can be distinguished from $Q_{2^{r}}$ by the degree $[\mathbb{Q}(\xi): Q]$ of the center field. Finally, note that $f_{E}=\left[\widehat{\mathbb{Q}}_{2}\left(\rho_{E}\right): \widehat{\mathbb{Q}}_{2}\right]$ is odd precisely when $E$ is non-split.

\section{References}

1. Bak, A.: Induction for finite groups revisited, J. of Pure and Applied Algebra 104, 235-241 (1995)

2. Berz, G.: Permutationsbasen für endliche Gruppen, Ph.D. thesis, Augsburg, 1994

3. Curtis, C. W., and Reiner, I.: "Methods of Representation Theory with Applications to Finite Groups and Orders, Volume II", John Wiley \& Sons, New York, 1987

4. Dress, A. W. M.: Contributions to the theory of induced representations, "Classical Algebraic $K$-Theory and Connections with Arithmetic" (H. Bass, ed.), Lecture Notes in Math., vol. 342, pp. 183-240, Springer-Verlag, New York, 1972

5. Dress, A. W. M.: Induction and structure theorems for orthogonal representations of finite groups, Ann. of Math. 102, 291-325 (1975)

6. Fontaine, J. M. : Sur la decomposition des algebres des groupes, Ann. Sci. Ecole Norm. Sup. 4, 121-180 (1971)

7. Goldschmidt, D. M., and Isaacs, I. M. : Schur indices in finite groups, J. of Algebra 33, 191-199 (1975)

8. Hambleton, I. , and Madsen, I.: Actions of finite groups on $\mathbf{R}^{n+k}$ with fixed set $\mathbf{R}^{k}$, Can. J. Math. 38, 781-860 (1986)

9. Hambleton, I. , Taylor, L. R., and Williams, E. B.: Detection theorems for $K$-theory and $L$-theory, J. of Pure and Applied Algebra 63, 247-299 (1990)

10. Hambleton, I. , Taylor, L. R., and Williams, E. B., Induction theory, MSRI preprint, 1990

11. James, G. , and Kerber, A.: "The Representation Theory of the Symmetric Group", Encyclopedia of Math. and App., vol. 16, Addison-Wesley, Reading, Mass., 1981

12. Oliver, R.: "Whitehead Groups of Finite Groups", L.M.S. Lecture Note Series, vol. 132, Cambridge Univ. Press, Cambridge, 1988

13. Reiner, I.:"Maximal Orders", L.M.S. Monographs, vol. 5, Academic Press, London, 1975

14. Ritter, J.: Ein Induktionssatz für rationale Charaktere von nilpotenten Gruppen, Journal f. reine u. angew. Math. 254, 133-151 (1972)

15. Roquette, P.: Realisierung von Darstellungen endlicher nilpotenter Gruppen, Archiv. der Math. 9, 241-250 (1958)

16. Segal, G.: Permutation representations of finite $p$-groups, Quart. J. Math. Oxford (2) 23, 375-381 (1972)

17. Serre, J. P.: "Linear Representations of Finite Groups", Graduate Texts in Math., vol. 42, Springer-Verlag, New York, 1977

18. Serre, J. P.: "Local Fields", Graduate Texts in Math., vol. 67, Springer-Verlag, New York, 1979 